\title{
THz diffractive focusing structures for broadband application
}

\author{
K. Liebert, ${ }^{* 1}$ M. Rachon, ${ }^{1}$ J. Bomba, ${ }^{1}$ A. Sobczyk, ${ }^{1}$ P. Zagrajek ${ }^{2}$, M. Sypek ${ }^{1}$, J. Suszek, ${ }^{1}$ and A. Siemion ${ }^{1}$ \\ ${ }^{I}$ Faculty of Physics, Warsaw University of Technology, 75 Koszykowa Str., 00-662 Warsaw, Poland \\ ${ }^{2}$ Institute of Optoelectronics, Military University of Technology, 2 Urbanowicz Str., 00-908 Warsaw, Poland
}

Received September 08, 2018; accepted September 30, 2018; published September 30, 2018

\begin{abstract}
Analysis of optical structures that can work for a broadband range of $\mathrm{THz}$ radiation is the aim of this article. Such structures can be designed as kinoforms of higher order or elements with an extended depth of focus, like axicons or light sword elements. A theoretical and experimental comparison of different optical elements for three significantly different frequencies is performed.
\end{abstract}

$\mathrm{THz}$ radiation possesses interesting features that make it desirable for security applications [1]. THz scanners [2] are already used at the airports and can detect concealed, dangerous items. This radiation is not ionizing, therefore, it is safe for living organisms [3-4] as opposed to commonly used X-rays. Lately, the $\mathrm{THz}$ postal scanner [5] has been demonstrated and enabled examination of the content of an envelope without opening it. Moreover, it is possible to determine whether the envelope does not contain any dangerous objects made of metal, wood, plastic or disturbing powders, but requires scanning with more than one frequency. For this reason, a lens is desirable, capable to shape the beam for few specific wavelengths that are drastically different (for example for $\left.\frac{\lambda}{2}, \lambda, 2 \lambda\right)$.

The main drawback is that those $\mathrm{THz}$ detectors existing on the market have a low signal to noise ratio [6] and thus it is necessary to accurately focus incident radiation. In this case, beam shaping seems to be necessary and can be carried out by mirrors [7], refractive structures [8] or structures based on the phenomenon of diffraction [9]. Diffractive optical elements (DOEs) are characterized by lightness, small thickness and relatively low price.
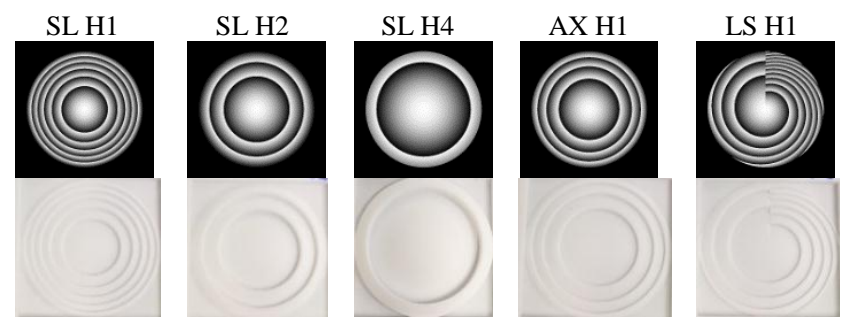

Fig. 1 Phase distribution of designed optical elements (upper row) corresponding to the depth map, where black introduces 0 and white $2 p \pi$ phase shift, where $p=1,2$ or 4 and is the order of the kinoform. The photograph of 3D printed optical elements (lower row).

*E-mail: karolina.liebert@ gmail.com
In this article DOEs working at different frequencies will be discussed - first, the comparison of spherical lens (SL) coded as kinoform [10] and high order kinoforms (HOKs) [11-12] and then elements with extended depth of focus axicons (AX) [13] and light sword elements (LS) [14] (as $1^{\text {st }}$ order kinoform structures).

Kinoform structures, due to their maximal phase shift of only $2 \pi$, are for narrowband use only, because with illumination of different wavelengths than the design wavelength (DWL) they are characterized by chromatic aberration. A different approach to coding phase distribution is using high order kinoforms that combine the advantages of refractive and diffractive structures. This type of kinoform has the phase shift several times greater than $2 \pi$. For this reason, it has achromatic properties like a refractive structure while retaining the advantages of a diffractive structure. Despite the fact that HOKs are several times thicker than first order kinoforms, they are still significantly thinner and lighter than refractive ones. However, the simulation algorithm should assume this thickness (corresponding to multiple wavelengths) and thus the mBPM (modified Beam Propagation Method) [15] is applied.

Table. 1. Formulas describing the phase distribution of designed optical elements. Here, $f$ is the designed focal length equal to $50 \mathrm{~mm}, \lambda$ is the wavelength $(\mathrm{DWL}=1 \mathrm{~mm}), a=0.028, d_{2}=90, f_{1}=40, f_{2}=80$.

\begin{tabular}{|l|c|}
\hline Element & Phase distribution $\phi$ \\
\hline SL H1 & $-\frac{2 \pi}{\lambda} \sqrt{r^{2}+f^{2}}$ \\
\hline SL H2 & $-\frac{\pi}{\lambda} \sqrt{r^{2}+f^{2}}$ \\
\hline SL H4 & $-\frac{\pi}{2 \lambda} \sqrt{r^{2}+f^{2}}$ \\
\hline AX H1 & $-\frac{\pi}{\lambda a} \ln \mid 2 a \sqrt{a^{2} r^{4}+\left(1-2 a d_{2}\right) r^{2}+d_{2}^{2}}+2 a^{2} r^{2}$ \\
\hline LS H1 & $-\frac{2 \pi}{\lambda} \sqrt{r^{2}+\left(f_{1}+\frac{f_{2} \Theta}{2 \pi}\right)^{2}}-\frac{2 \pi}{\lambda}\left(f_{1}+\frac{f_{2} \Theta}{2 \pi}\right)$ \\
\hline
\end{tabular}

Another method of reducing chromatic aberrations is using structures that increase the depth of focus (like LS and AX structures). It can result in focusing all considered wavelengths in the same way into some line 
segment along the optical axis. Thus, chromatic aberration will be reduced.

All optical elements were calculated in a non-paraxial approach. Phase distributions $\phi$ presented in the greyscale as depth maps (described with formulas given in Tab. 1) and photos of the designed structures are shown in Fig. 1.

Computer modeling for all structures was carried out (using Light Sword 6.0 software available in the Laboratory of Optical Information Processing) on arrays $4096 \times 4096$ pixels with the sampling $117 \mu \mathrm{m} \times 117 \mu \mathrm{m}$ (which was determined by the resolution of a $3 \mathrm{D}$ printing machine). The structures were designed for the wavelength $\mathrm{DWL}=1 \mathrm{~mm}$. However, simulations were carried out also for: $0.5 \mathrm{~mm}(600 \mathrm{GHz})$ and $2 \mathrm{~mm}$ (150GHz). The absorption coefficient strongly depended on the frequency (using polyamide 12 material was assumed) and was equal to: $0.5 \mathrm{~cm}^{-1}$ (@150GHz), $1 \mathrm{~cm}^{-1}$ (@300GHz) and $7 \mathrm{~cm}^{-1}$ (@600GHz), while the refractive index was equal to 1.59 for these frequencies.

The results of computer modeling compared to experimental evaluation are presented in Tables 2 and 3.

a)

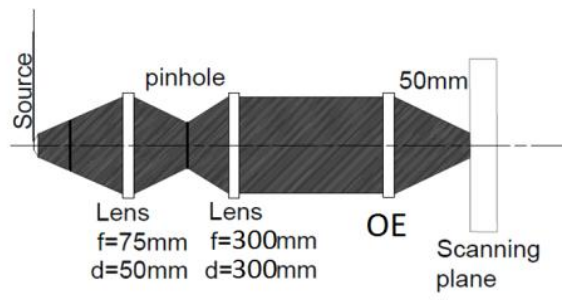

b)

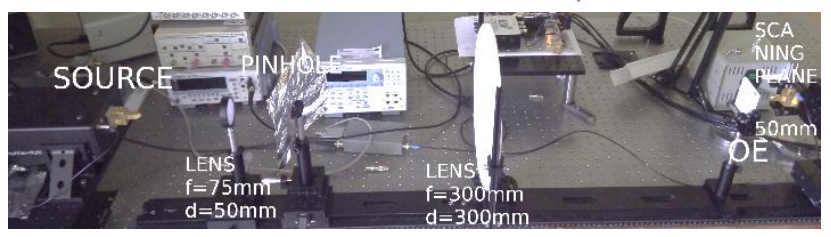

Fig. 2. The optical system in the experiment: a) a scheme and b) a photo.

The experimental optical system is presented in Fig. 2. $\mathrm{THz}$ radiation was provided by a VDI (Virginia Diodes, Inc., Charlottesville, VA, USA) frequency multiplier and illuminated a lens $(f=75 \mathrm{~mm}, d=50 \mathrm{~mm})$ that focused the incident light on a circular aperture with a diameter of $2 \mathrm{~mm}$. Then, at a distance of $300 \mathrm{~mm}$ after pinhole a HDPE lens $(f=d$ of $300 \mathrm{~mm}$ ) was placed forming a quasi-plane wave that illuminated the designed optical element (OE). Finally, the image was scanned at a distance of $50 \mathrm{~mm}$ behind the structure (OE).

Measurements were performed for three frequencies: $150 \mathrm{GHz}, 300 \mathrm{GHz}$ and $600 \mathrm{GHz}$. The power of generated radiation was equal to: $1 \mathrm{~mW}, 0.8 \mathrm{~mW}$, and $40 \mu \mathrm{W}$, respectively. The emitters were equipped with horn antennas which formed divergent beams.

The focal point was scanned with a Schottky diode detector also equipped with a horn antenna whose aperture diameter was equal to: $8.4 \mathrm{~mm}, 5.6 \mathrm{~mm}$ and $2.4 \mathrm{~mm}$ for frequencies $150 \mathrm{GHz}, 300 \mathrm{GHz}$ and $600 \mathrm{GHz}$, respectively. The scanning step of the detector was $2 \mathrm{~mm}$. To measure the signal from the detector, a lock-in system (Stanford Research Systems SR830) was used, based on modulation at $187 \mathrm{~Hz}$.

Table 2. Intensity distributions being the results of computer modeling and experimental results of a spherical lens coded as a kinoform of first, second and fourth order. The size of the area is equal to $30 \mathrm{~mm}$.

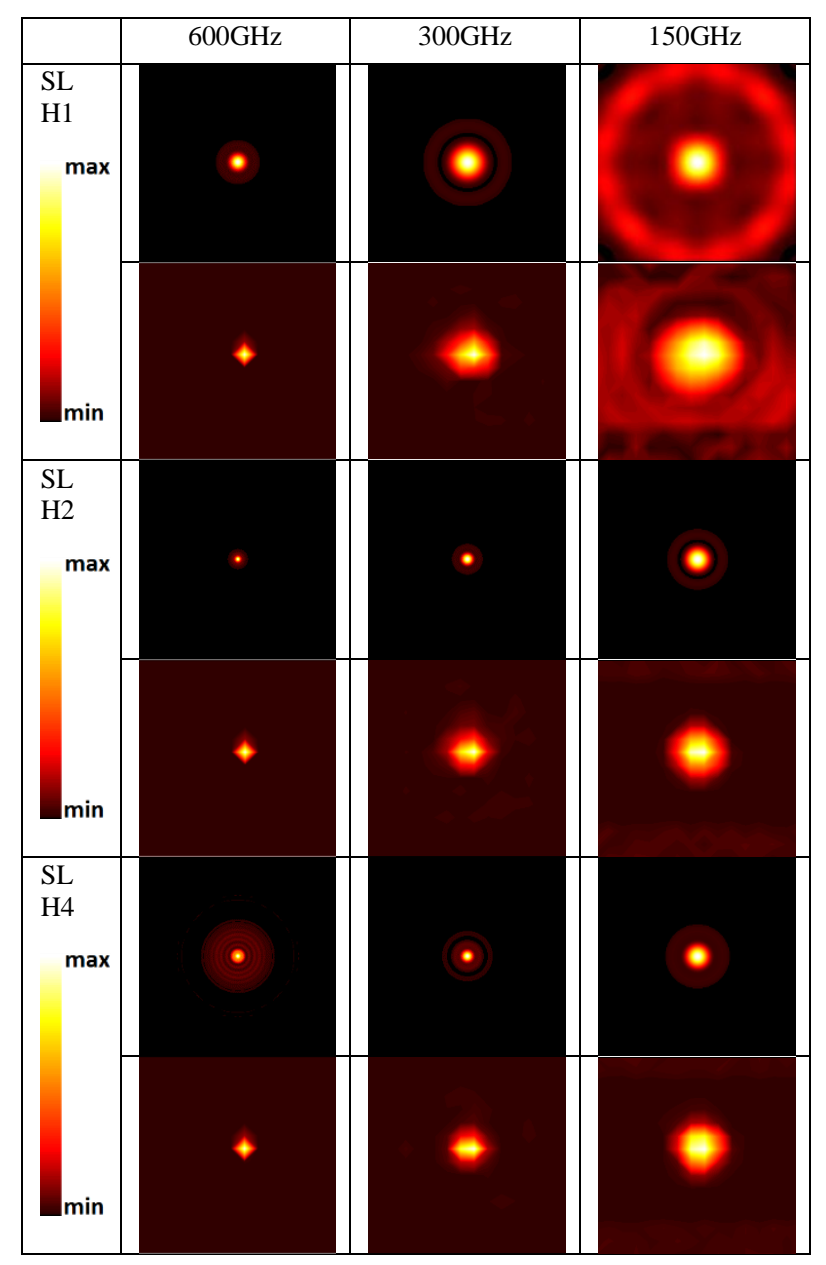

All optical elements were manufactured using selective laser sintering (SLS) as a 3D printing method [16], using a polyamide 12 (PA12) material. The refractive index of PA12 for all frequencies is approximately equal to 1.59 , which results in the thickness of the structure $1.69 p \mathrm{~mm}$, where $p=1,2$ or 4 and is the order of the kinoform.

The experimental results showing intensity distribution are presented in Tab. 2 and Tab. 3. Due to the fact that different power of sources and different detectors for different wavelengths were used, the maximal value in the focal spot in the experimental part should not be compared between frequencies. Taking into account the fact that the scanning step was $2 \mathrm{~mm}$, we verified for each frequency if the relation between measured intensity 
values for different elements were corresponding to the values calculated in numerical simulations. Any deviation of measured values from the simulation results corresponded to the sampling difference and relatively large scanning step. Radiation incoming to the detector could not be registered efficiently due to a different place and angle of the incident at this position beam.

It should be noticed that a smaller diameter of the focal spot for higher frequency corresponds to the theory describing the Airy disc size (proportional to the wavelenght). Moreover, SL designed as a kinoform of $1^{\text {st }}$ order should not work for the frequency $150 \mathrm{GHz}$, which can be observed in Tab. 2.

Table 3. Intensity distributions being the results of computer modeling and experimental evaluation for axicon (AX) and light sword optical element (LS). The size of the area is equal to $30 \mathrm{~mm}$.

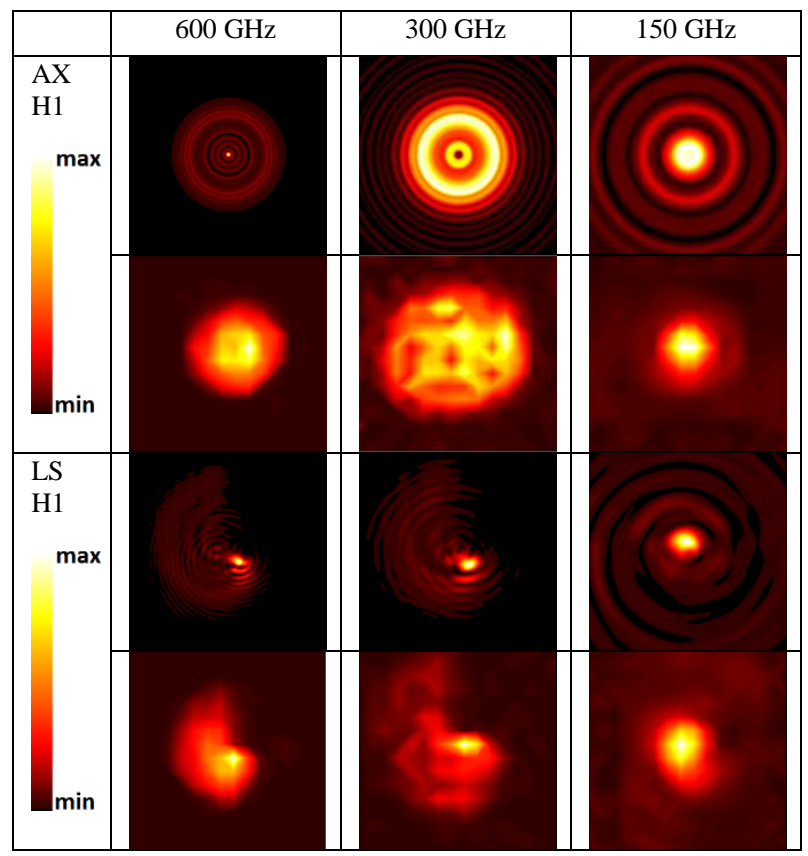

In conclusions, the main issue was to investigate if the designed optical elements: spherical lenses (as kinoforms of $2^{\text {nd }}$ and $4^{\text {th }}$ order), light sword optical element (LS) and axicon (AX) have better optical properties (focal spot and maximum intensity) then the Fresnel lens (spherical lens as a kinoform of $1^{\text {st }}$ order). Each of the tested structures is characterized by better performance at $150 \mathrm{GHz}$ than SL H1 (except SL H4, which is related to a very high attenuation coefficient of PA12 material at $600 \mathrm{GHz}$ (both in computer modeling and in the experiment).

The axicon structure itself and experimental setup should be redesigned. Here, probably only the main zone was illuminated and therefore the nature of the structure is not maintained.
This study was supported by project 2017/25/N/ST7/02757 from National Science Centre, Poland and partially supported by the National Center for Research and Development in Poland grant LIDER/020/319/L-5/13/NCBR/2014.

\section{References}

[1] M.C. Kemp, P.F. Taday, B.E. Cole, J.A. Cluff, A.J. Fitzgerald, W.R. Tribe, International Society for Optics and Photonics 5070, 44 (2003). https://doi.org/10.1117/12.500491

[2] D. Yavorskiy, J. Marczewski, K. Kucharski et al., Phot. Lett. Poland 4(3), 100 (2012). http://photonics.p1/PLP/index.php/letters/article/view/4-36

[3] A.A. Angeluts, A.B. Gapeyev, M.N. Esaulkov et al., Quant. Electr. 44(3), 247 (2014). http://iopscience.iop.org/article/10.1070/QE2014v044n03ABEH01533 7

[4] Q. Sun, Y. He, K. Liu, S. Fan, E.P. Parrott, E. Pickwell-MacPherson, Quant. Imag. Med. Surg. 7(3), 345 (2017). http://qims.amegroups.com/article/view/15326/15541

[5] J. Suszek, A. Siemion, M. Bieda et al., IEEE Thz Sci. T. 5, 15326 (2015). http://qims.amegroups.com/article/view/15326/15541

[6] M. Naftaly, R. Dudley, Opt. Lett. 34(8), 1213 (2009). https://www.osapublishing.org/ol/abstract.cfm?uri=ol-34-8-1213

[7] C. Bruckner, G. Notnia, A. Tünnermann, Optik 121, 1 (2010). https://www.sciencedirect.com/science/article/pii/S0030402608001393 ?via\%3Dihub

[8] J. Richter, A. Hofmann, L.-P. Schmidt, Proc. 31st Europ. Microw. Conf., London 2001. http://ieeexplore.ieee.org/document/4140002/

[9] E.D. Walsby, S. M. Durbin, D.R S. Cumming, R.J. Blaikie, Curr. App. Phys. 4, 102 (2004) https://www.sciencedirect.com/science/article/pii/S1567173903001524

[10] J.A. Jordan Jr. et al, Appl Opt. 9(8), 1883 (1970). https://doi.org/10.1364/AO.9.001883

[11] J.C. Marron, D.K. Angell, A.M. Tai, Intern. Soc. Opt. Photon. 1211, 62 (1990). https://doi.org/10.1117/12.17930

[12] J. Suszek, A.M. Siemion, N. Błocki, M. Makowski, A. Czerwiński, J. Bomba, P. Zagrajek et al., Opt. Expr. 22(3), 3137 (2014). https://doi.org/10.1364/OE.22.003137

[13] J. Sochacki, A. Kołodziejczyk, Z. Jaroszewicz, S. Bara, App. Opt. 31(25), 5326 (1992). https://doi.org/10.1364/AO.31.005326

[14] A. Kołodziejczyk, S. Bará, Z. Jaroszewicz, M. Sypek, J. Modern Opt. 37(8), 1283 (1990). https://doi.org/10.1080/09500349014551431

[15] M. Sypek, Opt. Commun. 116, 43 (1995). https://doi.org/10.1016/0030-4018(95)00027-6

[16] J.P. Kruth, X. Wang, T. Laoui, L. Froyen, Assembly Autom. 23(4), 357 (2003). https://doi.org/10.1108/01445150310698652 